\title{
PROBLEMS ASSOCIATED WITH THE M-DWARF POPULATION
}

\author{
M. SCHMIDT \\ Hale Observatories, \\ California Institute of Technology, Carnegie Institution of Washington, \\ Pasadena, Calif., U.S.A.
}

\begin{abstract}
The existence of a rich population of late M-type dwarfs with small peculiar velocities is discussed on the basis of the work of Weistrop, Murray and Sanduleak, Pesch and others. These stars probably supply the 'missing' mass in the solar neighborhood. Their small peculiar velocities create problems related to the stability of the galactic disk.
\end{abstract}

Observations seem to indicate that there exists in the solar neighborhood a dense layer of M-type dwarfs with small peculiar velocities. These stars would solve the longstanding problem of the 'missing mass'. However, their small peculiar velocities create problems so severe that theoreticians would prefer these stars not to exist. I wish to review the observations pertaining to the space density and the kinematics of the M-type dwarfs, and to discuss briefly the theoretical problems.

The first evidence for a large population of M-type dwarfs came from an objective prism survey by Sanduleak (1964). He found 1200 M-type stars over an area of 120 sq deg and used colors and magnitudes to derive a space density of M-type dwarfs that was three times that corresponding to the stellar luminosity function of Luyten (1938). These results did not attract wide attention since they were published in summary form as part of the Annual Report of the Warner and Swasey Observatory.

Several years later Donna Weistrop (1972a, b) undertook a program of star counts as a function of color in a field near the north galactic pole. She derived a luminosity function that shows an increasing excess over the luminosity function of Van Rhijn (1936), to a factor of about 10 at $M_{v}=13$. Table I reproduces a table given by Veeder (1974) based on Weistrop's luminosity function. The total mass density of mainsequence stars is $0.16 M_{\odot} \mathrm{pc}^{-3}$ if we adopt the estimated values of the luminosity

TABLE I

Local mass densities of dwarfs

\begin{tabular}{llll}
\hline$M_{v}$ & $\begin{array}{l}n \\
\left(\mathrm{pc}^{-3}\right)\end{array}$ & $M / M_{\odot}$ & $\begin{array}{l}\varrho \\
\left(M_{\odot} \mathrm{pc}^{-3}\right)\end{array}$ \\
\hline$<9$ & & & 0.018 \\
10 & 0.01 & 0.45 & 0.005 \\
11 & 0.03 & 0.35 & 0.010 \\
12 & 0.06 & 0.27 & 0.016 \\
13 & 0.13 & 0.20 & 0.026 \\
14 & $0.25^{\mathrm{a}}$ & 0.16 & $0.040^{\mathrm{a}}$ \\
15 & $0.40^{\mathrm{a}}$ & 0.12 & $0.048^{\mathrm{a}}$ \\
\hline
\end{tabular}


function beyond $M_{v}=13$ as given in Table I. Since interstellar gas contributes about $0.03 M_{\odot} \mathrm{pc}^{-3}$ and white dwarfs around $0.02 M_{\odot} \mathrm{pc}^{-3}$ we can apparently account for the dynamical value of the local mass density $\left(0.21 M_{\odot} \mathrm{pc}^{-3}\right)$ derived by Oort (1965) on the assumption that the 'missing mass' is distributed like the interstellar gas. Weistrop found, in fact, that the density distribution of the fainter stars resembles that of interstellar gas. The corresponding scale height of only about $110 \mathrm{pc}$ suggests that these stars have peculiar velocities of only around $10 \mathrm{~km} \mathrm{~s}^{-1}$.

I would like to illustrate the observational evidence for such a large number of late M-type dwarfs by deriving their expected numbers on the basis of Van Rhijn's luminosity function. We use an exponential density distribution $D(z) \sim \exp \left(-z / z_{s}\right)$ with a scale height $z_{s}$ of $300 \mathrm{pc}$, which represents approximately Weistrop's $D(z)$ for $\mathrm{G}$ and K-type dwarfs. Mean absolute visual magnitudes $M_{t}$ as a function of color $(B-V)$ based on available trigonometric parallaxes (see Weistrop, 1972b, for references) are given in Table II. These absolute magnitudes are based on apparent mag-

TABLE II

Observed and predicted star counts

\begin{tabular}{|c|c|c|c|c|c|c|c|c|c|}
\hline \multirow[t]{2}{*}{$\begin{array}{l}B-V \\
M_{t} \\
M_{0} \\
\text { Spect. type }\end{array}$} & $\begin{array}{l}1.40-1 \\
9.5 \\
10.0 \\
\text { dM0- }\end{array}$ & & & $\begin{array}{l}1.50-1 \\
11.0 \\
11.5 \\
\text { dM4 }\end{array}$ & & & $\begin{array}{l}1.60-1 \\
12.5 \\
13.0 \\
\text { dM5 }\end{array}$ & & \\
\hline & Obs. & Pred. & $\frac{\text { Obs. }}{\text { Pred. }}$ & Obs. & Pred. & $\frac{\text { Obs. }}{\text { Pred. }}$ & Obs. & Pred. & $\frac{\text { Obs. }}{\text { Pred }}$ \\
\hline $\begin{array}{r}M_{v}=13.5 \\
14.5\end{array}$ & $\begin{array}{r}4 \\
15\end{array}$ & $\left.\begin{array}{r}3 \\
12\end{array}\right\}$ & 1.3 & $\begin{array}{r}9 \\
13\end{array}$ & $\left.\begin{array}{l}0.7 \\
2.7\end{array}\right\}$ & 6 & $\begin{array}{l}2 \\
9\end{array}$ & $\left.\begin{array}{l}0.1 \\
0.4\end{array}\right\}$ & 22 \\
\hline 15.5 & 37 & 37 & 1.0 & 20 & 10 & 2 & 27 & 1.6 & 17 \\
\hline 16.5 & 70 & 83 & 0.8 & 68 & 32 & 2 & 54 & 6 & 9 \\
\hline 17.5 & 133 & 254 & 0.5 & 108 & 97 & 1 & 79 & 20 & 4 \\
\hline
\end{tabular}

nitude-selected samples. The mean absolute magnitude in a volume of space $M_{0}$ is fainter by $\sigma^{2}(A(m))^{-1}(\mathrm{~d} A(m) / \mathrm{d} m)$ magnitudes (Malmquist, 1927). For $\sigma= \pm 0.6 \mathrm{mag}$. the Malmquist correction amounts to $0.5 \mathrm{mag}$. We adopt a gaussian distribution of absolute magnitude around $M_{0}$ with a dispersion of $0.75 \mathrm{mag}$., to take into account the variation of the mean absolute magnitude within the interval of $B-V$. We used a classical $(m, \log r)$ table technique (Schouten, 1914) to derive the predicted star counts.

Table II gives the predicted star counts as well as those observed by Weistrop over an area of $13.5 \mathrm{sq}$ deg in three $B-V$ color ranges. The results can be summarized in two conclusions: (1) At bright apparent magnitudes, $m_{v} \simeq 14$, the observations show an excess that increases steeply with color; while for $B-V=1.45$ there is little discrepancy, we find the ratio observed/predicted to be a factor of 6 for $B-V=1.55$, and a factor of 20 for $B-V=1.65$, and (2) the ratio observed/predicted decreases for increasing apparent magnitude at a given color. Conclusion (1) suggests that the actual luminosity function shows an increasing excess over Van Rhijn's function for 
$B-V>1.5$ or $M_{v}>11$, while (2) indicates that the scale height for these stars is smaller than the assumed value of $300 \mathrm{pc}$. These conclusions, based on a direct confrontation of the expected and observed numbers in Table II, are qualitatively similar to those obtained by Weistrop.

The main uncertainty in the densities is related to the absolute magnitudes. If we adopt for the three color ranges of Table II absolute magnitudes $M_{0}$ of 10,11 , and 12 instead of $10,11.5$, and 13, respectively, then the predicted star counts would change by factors of $\frac{2}{3}, \frac{4}{3}$, and $\frac{8}{3}$, respectively. Apparently such a substantial revision of the absolute magnitude calibration would do little to explain observed excesses in the counts by factors of 6 and 20 , for $B-V=1.55$ and 1.65 , respectively.

An entirely different approach was taken by Murray and Sanduleak (1972) who investigated proper motions of 21 Sanduleak survey stars near the galactic north pole. They found: (1) none of the stars could be giants since all had distinctly nonzero motions, (2) the dispersion of velocities parallel to the galactic plane corresponds to about $\pm 10 \mathrm{~km} \mathrm{~s}^{-1}$, and (3) the mean parallax together with the statistics of the Sanduleak stars results in a number density of 0.23 stars per cubic parsec down to $M_{v}=13$, in good agreement with Weistrop's luminosity function.

The small velocity dispersion found by Murray and Sanduleak is compatible with the small vertical scale height derived by Weistrop. The derivation of the space density from the proper-motion work has been done in a somewhat approximate fashion and I believe that the results based on the star counts deserve rather more weight. Nevertheless, the agreement between the two investigations is remarkable.

Pesch (1972) undertook a spectroscopic study of M stars from the Sanduleak objective prism survey. He considered only stars that showed no proper motion larger than 0.05 per year. Only two of the 17 stars fainter than $V=12.5$ were found to be giants. The published $B-V$ colors allow an estimate of absolute magnitude and distance for each of the dwarfs. I find that the 12 dwarfs within 100 pc have transverse velocities less than around $15 \mathrm{~km} \mathrm{~s}^{-1}$. These no-proper-motion stars constitute about half of the Sanduleak stars according to Pesch. All these results are compatible with those of Murray and Sanduleak.

The transition from the large to the small scale height appears to occur at a $B-V$ color of around 1.50. The corresponding spectral type of M2 or M3 is somewhat uncertain since colors and spectral types do not correlate well for early M-type dwarfs. Few stars later than M3 are contained in the McCormick objective-prism survey (Vyssotsky, 1956, and earlier papers) and this may explain the apparent discrepancy noted by Gliese (1974) between the velocity dispersions of the Pesch stars and the McCormick stars. Similarly, the McCarthy-Treanor (1964) objective-prism survey of a region near the Pleiades contains no stars later than M3. Hence, the relatively large velocity dispersions between 17 and $32 \mathrm{~km} \mathrm{~s}^{-1}$ derived by B. F. Jones (1972) for $M$ stars in this survey may be understood.

D. H. P. Jones (1973) has carried out a photoelectric narrow-band classification of M-type stars found in an objective-prism survey near the south galactic pole by McCarthy et al. (1964). He finds a space density only one-fifth of that found by 
Murray and Sanduleak, and derives a dispersion of peculiar velocities of $\pm 36 \mathrm{~km} \mathrm{~s}^{-1}$. The disagreement with the results of Weistrop and of Murray and Sanduleak is remarkable. B. F. Jones (1972) had noted that the McCarthy Pleiades survey is only one-third complete. If this applies to the South Pole survey too, then a substantial correction is in order. As noted by D. H. P. Jones (1973) the McCarthy stars are concentrated to early $M$ subtypes whereas the Sanduleak stars have a more even distribution of subtype. Hence, the McCarthy survey may be particularly incomplete for later types, which are the ones that show a high density and small peculiar velocities.

I have not considered in this review investigations based on catalogs of stars with large proper motion since these carry little information about the slow-moving late M-type dwarfs.

The total evidence points to the existence of a rich population of late M-type dwarfs with small velocity dispersion. The small velocity dispersion leads to two problems, however. The first problem concerns the age of these objects. It has been generally assumed that the large velocity dispersion of the K-type dwarfs is caused by gravitational encounters with large gas clouds (Spitzer and Schwarzschild, 1953) or perhaps density waves connected with spiral structure. If the late M-type dwarfs are as old as the K-type dwarfs they should have suffered the same dynamical history. The discrepancy in the velocity dispersion suggests that the late M-type dwarfs are much younger, perhaps around one billion years. However, these stars carry two-thirds of the local mass density and it seems inconceivable that so large a fraction of the interstellar gas condensed into stars as recent as one billion years ago. Hence, we have to assume that the Spitzer-Schwarzschild mechanism does not operate and then we have to find a new explanation for the observed increase in the velocity dispersion along the main sequence from types $\mathrm{A}$ to $\mathrm{K}$.

The second problem arising from the existence of the slow-moving M-type dwarfs concerns the stability of the disk against axi-symmetric perturbations. For a surface density of around 60 solar masses per $\mathrm{pc}^{2}$ the stability criterion established by Toomre (1964) requires a velocity dispersion of at least $28 \mathrm{~km} \mathrm{~s}^{-1}$. This is much higher than the observed value around $10 \mathrm{~km} \mathrm{~s}^{-1}$ and as shown by P. Biermann in the preceding communication there is no possibility to satisfy this criterion with a mixture of populations.

In summary, the solution of one problem (that of the missing mass) has created two new problems. Further studies, both observational and theoretical, are called for. Perhaps in this confrontation between observation and theory, new insights in the nature and evolution of our Galaxy will be generated eventually.

\section{References}

Gliese, W.: 1974, Astron. Astrophys. 34, 147.

Jones, B. F.: 1972, Monthly Notices Roy. Astron. Soc. 159, 3P.

Jones, D. F. P.: 1973, Monthly Notices Roy. Astron. Soc. 167, 19 P.

Luyten, W. J.: 1938, Publ. Astron. Obs. Univ. Minnesota 2, No. 7. 
Malmquist, K. G. : 1927, Lund Obs. Medd., Ser. II, No. 46.

McCarthy, M. F., Bertiau, F. C., and Treanor, P. J.: 1964, Ricerche Astron. Vaticana 6, 571.

McCarthy, M. F. and Treanor, P. J.: 1964, Ricerche Astron. Vaticana 6, 535.

Murray, C. A. and Sanduleak, N.: 1972, Monthly Notices Roy. Astron. Soc. 157, 273.

Oort, J. H. : 1965, Stars and Stellar Systems 5, 455.

Pesch, P.: 1972, Astrophys. J. 177, 519.

Sanduleak, N. : 1964, Astron. J. 69, 720.

Schouten, W. J. A.: 1918, dissertation, Groningen University.

Spitzer, L. and Schwarzschild, M.: 1953, Astrophys. J. 118, 106.

Toomre, A.: 1964, Astrophys. J. 139, 1217.

Van Rhijn, P. J.: 1936, Publ. Kapteyn Astron. Lab. Groningen, No. 47.

Veeder, G. J.: 1974, Astrophys. J. Letters, 191, L57.

Vyssotsky, A. N.: 1956, Astron. J. 61, 201.

Weistrop, D.: 1972a, Astron. J. 77, 366.

Weistrop, D. : 1972b, Astron. J. 77, 849.

\section{DISCUSSION}

Lecar: How does this data look expressed as number per unit mass (e.g. ascompared with Salpeter function $\left.n(m) \simeq m^{-2.3}\right)$ ?

Schmidt: The overall character of the luminosity function is like that of a Salpeter power law but there are systematic deviations as a function of mass, I believe.

Lecar: What masses are associated with $M_{0}=10,11.5,13$ ?

Schmidt: Of the order of half a solar mass.

Spitzer: Should one at least consider the possibility that these excess stars are not so far away as giants would be but somewhat further away than assumed? This assumption would place the stars somewhat above the main sequence and would give them a more nearly normal dispersion of velocities.

Schmidt: If these stars were well above the main sequence, then it would be surprising that they have not shown up in the parallaxe work, on which the relation between $M_{v}$ and $B-V$ is based.

King: Recently two Berkeley students, David Koo and Richard Kron, have determined radial velocities for 5 of the 21 Murray-Sanduleak stars. Their velocity dispersion is $10 \mathrm{~km} \mathrm{~s}^{-1}$. This tends to confirm the low velocity dispersion that has been suggested. In conjunction with the proper motions of Murray and Sanduleak, this also tends to confirm the conventional absolute magnitudes that they assumed. These stars have no $\mathrm{H} \alpha$ emission, which suggests that they are not young. Their spectra have dwarf characteristics.

Other programs are under way at Berkeley, extending Weistrop's study to fainter magnitudes, and measuring proper motions for the same stars. In using lists of stars found by spectral surveys, one should ask what spectral region was used. Blue surveys, like the McCormick survey, discriminate against late-M dwarfs, because of the steep spectral gradient at $\mathrm{Ca}$ I 4227, on which the classification depends. Sanduleak's survey was done in the red and was thus better able to pick up late M's. 